\title{
First Lotus aortic valve-in-valve implantation to treat degenerated Mitroflow bioprostheses
}

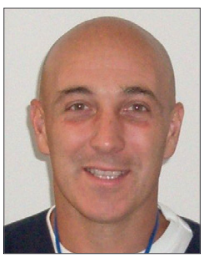

Fausto Castriota ${ }^{1 *}, \mathrm{MD}$; Caterina Cavazza ${ }^{1}, \mathrm{MD}$; Gioel Gabrio $\mathrm{Secco}^{2}, \mathrm{MD}$;

Antonio Micari ${ }^{1}$, MD; Alberto Cremonesi ${ }^{1}, \mathrm{MD}$

1. Cardiovascular Unit, Maria Cecilia Hospital, GVM Care \& Research, Cotignola, Italy; 2. Cardiovascular

Unit, SS Antonio e Biagio e Cesare Arrigo Hospital, Alessandria, Italy

This paper also includes supplementary data published online at: http://www.pcronline.com/eurointervention/97th_issue/297

\section{KEYWORDS}

- aortic valve disease

- transcatheter aortic valve implantation (TAVI)

- valve-in-valve

\section{Abstract}

Aims: Transcatheter aortic valve-in-valve implantation is an emerging alternative to conventional surgical aortic valve replacement (SAVR) in patients with degenerated aortic bioprostheses. This procedure presents a high risk of coronary occlusion, especially during treatment of patients implanted with a bioprosthesis with a lower distance between the leaflets and the coronary ostia, such as the Mitroflow valve (Sorin S.p.A., Milan, Italy). In this report we aim to describe the safety and feasibility of transfemoral valve-in-valve implantation of a new-generation Lotus ${ }^{\mathrm{TM}}$ Aortic Valve Replacement System (Boston Scientific, Marlborough, MA, USA) in a case of degenerated Mitroflow bioprostheses.

Methods and results: We describe a case of degenerated Sorin Mitroflow bioprostheses successfully treated with the transfemoral new-generation Lotus valve-in-valve system. During the procedure no coronary protection was required and the patient had no MACE at 30-day follow-up. To our knowledge, this is the first report of a Lotus valve implant in a degenerated Mitroflow prosthesis.

Conclusions: The transcatheter valve-in-valve procedure using the new-generation Lotus Valve System is a safe and feasible alternative to repeat cardiac surgery in high-risk patients with surgical degenerated Mitroflow bioprostheses. 


\section{Introduction}

Transcatheter aortic valve implantation (TAVI) is a valuable alternative to conventional surgical aortic valve replacement in highrisk patients with severe symptomatic aortic stenosis. While TAVI experience in native valves is rapidly progressing, recently TAVI has also evolved as a suitable and attractive option for highrisk patients with degenerated bioprostheses (valve-in-valve) $)^{1,2}$. However, this requires a careful patient selection and a thorough knowledge of the design, fluoroscopic appearances and implantation technique of the degenerated surgical bioprosthetic valve in situ, as well as its compatibility with currently available transcatheter valves. Moreover, the lateral displacement of the bioprostheses' leaflets might theoretically create a dangerous coronary ostia obstruction, especially in patients with a lower coronary ostia take-off and in those treated with particular biological bioprostheses, such as the Mitroflow (Sorin S.p.A., Milan, Italy). In fact, this bioprosthesis has an incidence of coronary obstruction eight times greater than other stented bioprostheses ${ }^{1,3}$, due to the lower distance between the leaflets mounted on the outside of the valve stent and the coronary ostia. The Mitroflow has been treated with balloon-expandable devices such as the Edwards SAPIEN (Edwards Lifesciences, Irvine, CA, USA) and self-expandable devices such as the Medtronic CoreValve ${ }^{\circledR}$ (Medtronic, Minneapolis, MN, USA), St. Jude Portico ${ }^{\mathrm{TM}}$ (St. Jude Medical, St. Paul, MN, USA), and the JenaValve (JenaValve Technology, Irvine, CA, USA) ${ }^{4}$. In this report, we describe a case of transfemoral valve-in-valve implantation using The Lotus ${ }^{\mathrm{TM}}$ Aortic Valve Replacement System (Boston Scientific, Marlborough, MA, USA), illustrating the advantage of its intrinsic repositionable/retrievable features.

\section{Case report}

We present a case of a 74-year-old man with hypertension, chronic obstructive pulmonary disease, moderate chronic renal failure (GFR $40 \mathrm{ml} / \mathrm{min}$ ) and previous PCI of the right coronary artery
(RCA). In 2009 he underwent surgical aortic valve replacement (Sorin Mitroflow n-23). In July 2014 he was re-admitted to our hospital for heart failure: the diagnostic work-up revealed a degenerated $23 \mathrm{~mm}$ Sorin Mitroflow aortic bioprosthesis with severe stenosis (peak/mean transvalvular gradient of 75/45 $\mathrm{mmHg}$ ) and moderate regurgitation. The calculated Society of Thoracic Surgeons (STS) predicted risk of mortality was 32.025\%, with a calculated logistic European System for Cardiac Operative Risk Evaluation I (EuroSCORE I) of 26.1\%. The multidisciplinary Heart Team reached consensus for transcatheter valve replacement in the failing aortic bioprosthesis. A computed tomographic angiography (CT scan) with $3 \mathrm{D}$ reconstruction was performed to assess the left ventricle outflow tract and sinotubular junction dimensions, the relative distance of the coronary ostia to the aortic valve annulus, the optimal angulation to implant the valve, and the iliac and femoral diameters. The CT scan showed a $13.8 \mathrm{~mm}$ height of the RCA ostium and a $7.7 \mathrm{~mm}$ height of the left main ostium from the Mitroflow ring (Figure 1). We decided to use a $23 \mathrm{~mm}$ diameter Lotus valve. The procedure was performed in our hybrid operating room, under local anaesthesia and mild sedation. A 7 Fr sheath was inserted in the left femoral artery (LFA) with a 0.018 " contralateral guidewire placed to protect the right femoral artery (RFA). An 18 Fr Lotus Introducer (Boston Scientific) was inserted into the RFA, and two ProGlide ${ }^{\circledR}$ systems were pre-implanted (Abbott Vascular, Santa Clara, CA, USA). After performing a baseline angiography (Figure 2A, Moving image 1), the $23 \mathrm{~mm}$ Lotus valve prosthesis was advanced through the Mitroflow sewing ring (Figure 2B, Moving image 2) and gradually deployed under fluoroscopic and angiographic guidance (Figure 2C, Figure 2D, Moving image 3), without ventricular pacing. During valve deployment, several angiographic controls were carried out to assess the patency of coronary ostia (Figure 2E, Moving image 4). Once device stability was confirmed and the position deemed optimal (Figure 2F), a final angiography was performed (Figure $\mathbf{2} \mathbf{G}$, Moving image 5 ) to confirm the
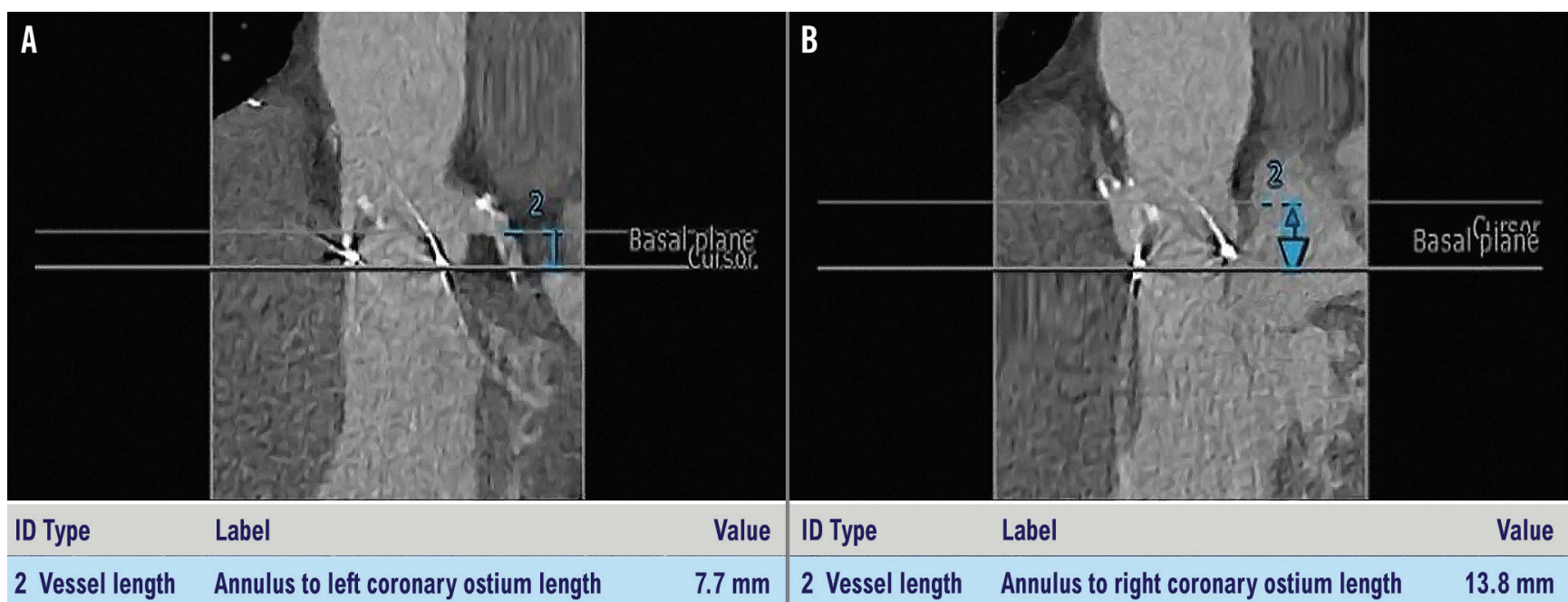

\begin{tabular}{|llr|llr}
\hline ID Type & Label & Value & ID Type & Label & Value \\
\hline 2 Vessel length & Annulus to left coronary ostium length & $7.7 \mathrm{~mm}$ & 2 Vessel length & Annulus to right coronary ostium length & $13.8 \mathrm{~mm}$
\end{tabular}

Figure 1. Height of LCA (A) and RCA (B) ostia from the Mitroflow ring calculated on the CT scan. 

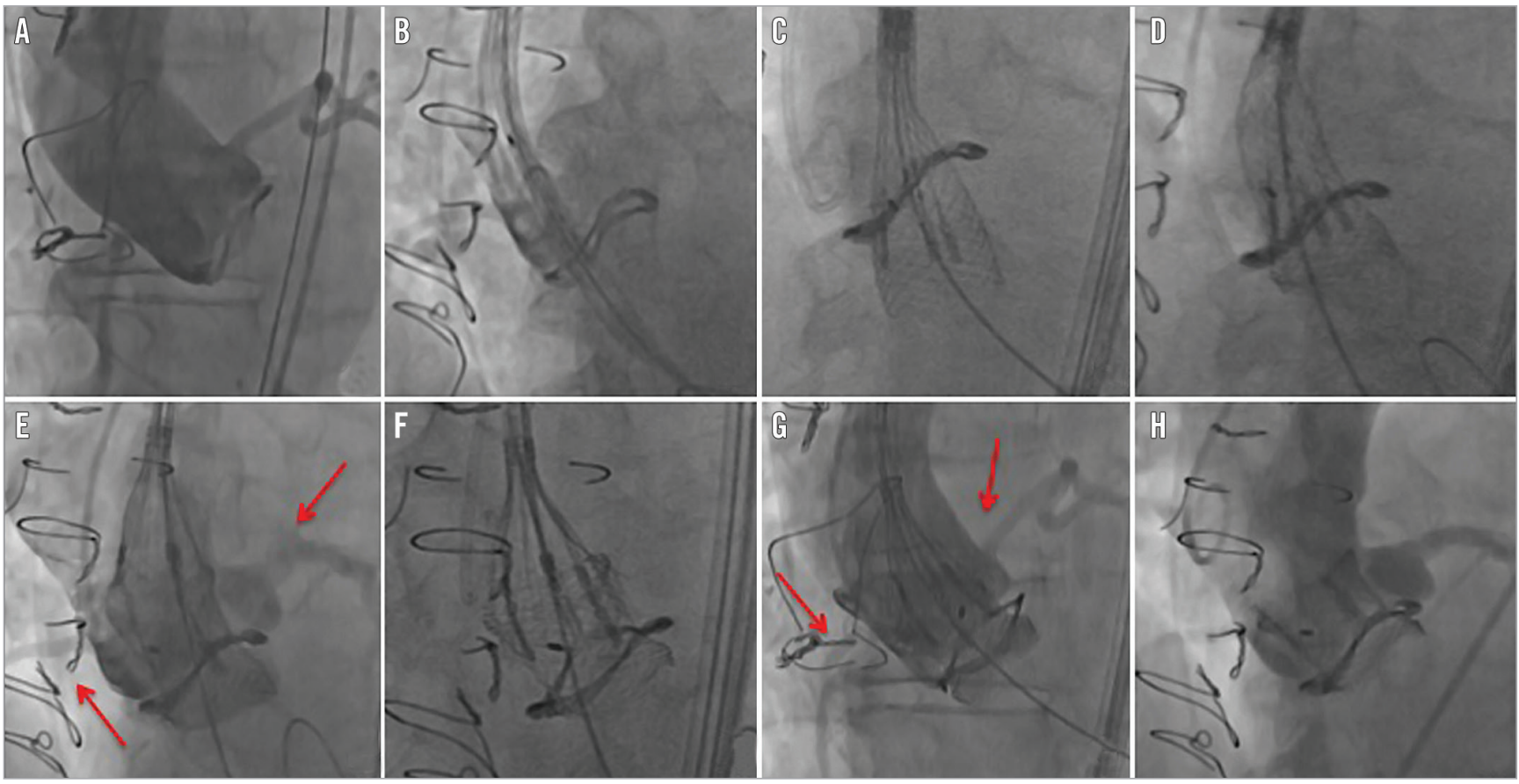

Figure 2. Step by step valve positioning. A) Baseline angiography. B) Lotus valve prosthesis crosses the Mitroflow sewing ring. C) \& D) The valve is gradually deployed under fluoroscopic and angiographic guidance; it shortens during deployment. E) Angiography to assess the patency of coronary ostia. F) Control of alignment of buckles and posts and continuous valve deployment. G) Final angiography to confirm the coronary patency and the absence of paravalvular leak. H) Final aortic root angiography.

coronary patency, and the valve was released definitively. The final aortic root angiography showed a correct position of the valve without paravalvular leak (Figure $\mathbf{2 H}$, Moving image 6), and there was no transvalvular gradient (Figure 3). Haemostasis was achieved by tying the ProGlide sutures while a crossover peripheral balloon was inflated in the right external iliac artery. The patient was discharged three days later. Post-procedural transthoracic echocardiography showed a well-performing valve and a peak/mean transvalvular gradient of $37 / 15 \mathrm{mmHg}$. At 30 days, the patient was asymptomatic, with a New York Heart Association (NYHA) functional Class I.

\section{Discussion}

The major finding of the clinical case we have described is that a transcatheter valve-in-valve procedure using the new-generation Lotus Valve System is safe and feasible in high-risk patients with surgical degenerated Mitroflow bioprostheses. The valve-in-valve is a challenging procedure due to the risk of patient-prosthesis mismatch, of malpositioning and of coronary occlusion. The first risk is frequently reported in patients with degenerated small bioprostheses $(<23 \mathrm{~mm})$ : implantation into $23 \mathrm{~mm}$ xenografts can be recommended only for patients with a body surface area less than $1.8 \mathrm{~m}^{2} .{ }^{5}$ In this reported case, the degenerated valve is a Mitroflow pericardial valve: this valvular system has an inner true diameter of $19 \mathrm{~mm}$, an outer diameter of $22.6 \mathrm{~mm}$ and a height of $14 \mathrm{~mm}$, so it might accommodate a $23 \mathrm{~mm}$ Lotus transcatheter valve. The sizing of the device is extremely important: undersizing increases the risk of paravalvular leak and/or migration/embolisation, while oversizing may lead to geometrical distortion of the transcatheter valve leaflets. The VIV International Data (VIVID) Registry reported an incidence of $3.5 \%$ of coronary obstruction ${ }^{1}$. Arguably, this phenomenon may be underestimated because coronary obstruction can be incomplete or mitigated by patent bypass grafts. The risk is increased during treatment of degenerated Mitroflow bioprostheses where the leaflets are mounted externally to the stent, thus

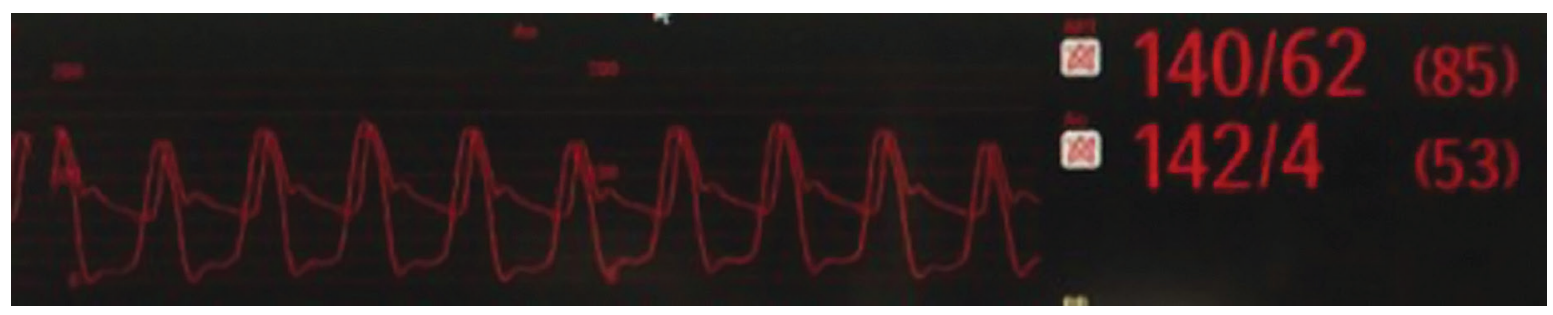

Figure 3. Post-procedural transvalvular gradient. 
resulting in a lower distance to the coronary ostia. The Lotus valve functions early in deployment, allows for a controlled mechanical opening with a radiopaque marker that guides a precise initial positioning, and it is completely repositionable and retrievable at any point, even at its maximal expansion, prior to release if required. Furthermore, the great advantage of this new-generation Lotus Valve System is that it allows assessment of coronary ostia patency at any point before definitive valve release. This is an improved safety profile of this new device, especially when treating a degenerated Mitroflow bioprosthesis or in patients with CT scan evidence of a low coronary take-off.

\section{Conclusions}

The transcatheter valve-in-valve procedure using the new-generation Lotus Valve System is a feasible and safe alternative to repeat cardiac surgery in high-risk patients with surgical degenerated Mitroflow bioprostheses. The possibility of valve repositioning, resheathing and retrieval offered by this new system minimises the risk of acute coronary ostia obstruction.

\section{Impact on daily practice}

Valve-in-valve treatment is a procedure with a high risk of coronary artery occlusion $(>15 \%)$, and in most cases in daily practice operators need to perform a coronary protection procedure before valve implantation. The percentage of coronary artery occlusion is higher with the Mitroflow valve because of the reasons we mentioned above. With the second-generation Lotus device, operators can assess coronary ostia patency during valve deployment, recapturing and repositioning the valve at any time during the procedure if required. This way the procedure can be performed without coronary artery protection even if the coronary take-off is very low, reducing the overall procedure time and risks.

\section{Conflict of interest statement}

F. Castriota is a proctor for the Lotus valve (Boston Scientific). The other authors have no conflicts of interest to declare.

\section{References}

1. Dvir D, Webb J, Brecker S, Bleiziffer S, Hildick-Smith D, Colombo A, Descoutures F, Hengstenberg C, Moat NE, Bekeredjian R, Napodano M, Testa L, Lefevre T, Guetta V, Nissen H, Hernandez JM, Roy D, Teles RC, Segev A, Dumonteil N, Fiorina C, Gotzmann M, Tchetche D, Abdel-Wahab M, DeMarco F, Baumbach A,
Laborde JC, Kornowski R. Transcatheter aortic valve replacement for degenerative bioprosthetic surgical valves: results from the global valve-in-valve registry. Circulation. 2012;126:2335-44.

2. Bapat V, Asrress KN. Transcatheter valve-in-valve implantation for failing prosthetic valves. EuroIntervention. 2014;10:900-2.

3. Dvir D, Leipsic J, Blanke P, Ribeiro HB, Kornowski R, Pichard A, Rodés-Cabau J, Wood DA, Stub D, Ben-Dor I, Maluenda G, Makkar RR, Webb JG. Coronary obstruction in transcatheter aortic valve-in-valve implantation: procedural evaluation, device selection, protection, and treatment. Circ Cardiovasc Interv. 2015;8. pii: e002079.

4. Conradi L, Kloth B, Seiffert M, Schirmer J, Koschyk D, Blankenberg S, Reichenspurner H, Diemert P, Treede H. The challenge of valve-in-valve procedures in degenerated Mitraflow bioprostheses and the advantage of using the JenaValve transcatheter heart valve. EuroIntervention. 2014;10:990-4.

5. Seiffert M, Conradi L, Baldus S, Knap M, Schirmer J, Franzen O, Koschyk D, Meinertz T, Reichenspurner H, Treede H. Impact of patient-prosthesis mismatch after transcatheter aortic valve-in-valve implantation in degenerated bioprostheses. J Thorac Cardiovasc Surg. 2012;143:617-24.

\section{Supplementary data}

Moving image 1. Baseline aortic root angiography showing a moderate aortic valve regurgitation.

Moving image 2. The valve advances through the Mitroflow sewing ring and is initially deployed in a very low position.

Moving image 3. After a gentle pullback, the valve is gradually deployed under fluoroscopic and angiographic guidance by rotating the delivery knob counter-clockwise. As it is designed to expand radially, the valve shortens during deployment.

Moving image 4. Control of the alignment of the buckles and posts, and angiography to assess the patency of coronary ostia prior to continuing valve deployment.

Moving image 5. The angiography shows absence of paravalvular leak and patency of both coronary ostia once the valve is locked (final position). Even at this point the valve is totally recapturable. Moving image 6. Final aortic root angiography shows a good angiographic result.

The supplementary data are published online at:

http://www.pcronline.com/

eurointervention/97th_issue/297 